\title{
Circulating microRNAs disclose biology of normal cognitive function in healthy elderly people - a discovery twin study
}

\author{
Jonas Mengel-From ${ }^{1,2}$ - Søren Feddersen ${ }^{3,4}$ - Ulrich Halekoh ${ }^{1}$ Niels H. H. Heegaard ${ }^{3,5}$ - Matt McGue ${ }^{1,6}$. \\ Kaare Christensen ${ }^{1,2,3} \cdot$ Qihua Tan $\mathbb{1}^{1,2} \cdot$ Lene Christiansen $^{1}$
}

Received: 2 June 2017 / Revised: 2 March 2018 / Accepted: 30 March 2018 / Published online: 2 May 2018

(c) European Society of Human Genetics 2018

\begin{abstract}
Neurobiology is regulated by miRNA. Here circulating plasma miRNAs were assayed on a 754 miRNA OpenArray platform using 90 monozygotic elderly twins (73-95 year of age) and associated with mini mental state examination (MMSE) and a five-component cognitive score (CCS) in an explorative study. Both ordinary individual and twin-pair analyses were performed with level of cognitive scores. Candidate miRNAs were further associated with cognitive decline over 10 years using up to six repeated assessments. A total of 278 miRNAs were expressed in plasma from at least ten participants and 23 miRNAs were nominally associated (i.e., at an uncorrected $p<0.05$ ) with CCS or MMSE in the paired analyses. Generally, elderly individuals with poor cognitive function had increase miRNA expression compared with equivalent individuals who performed better on the cognitive scale. Three miRNAs, miR-151a-3p, miR-212-3p and miR-1274b were associated with CCS both in the paired and the individual analysis. Four miRNAs found to be associated with CCS in cross-sectional analysis were also found to show an association in longitudinal analysis such that increase miRNA expression was associated with steeper cognitive decline. We propose a shared biological path underlies dementia and normative cognitive aging.
\end{abstract}

\section{Introduction}

Neurodegeneration is a major health impediment, particularly in the western world, where populations are aging and

Jonas Mengel-From

jmengel-from@health.sdu.dk

1 The Danish Aging Research Center and The Danish Twin Registry, Epidemiology, Biostatistics and Biodemography Unit, Department of Public Health, University of Southern Denmark, 5000 Odense, Denmark

2 Department of Clinical Genetics, Odense University Hospital, 5000 Odense, Denmark

3 Department of Clinical Biochemistry and Pharmacology, Odense University Hospital, 5000 Odense, Denmark

4 Department of Clinical Research, University of Southern Denmark, 5000 Odense, Denmark

5 Department of Autoimmunology \& Biomarkers, Statens Serum Institut, 2300 Copenhagen, Denmark

6 Department of Psychology, University of Minnesota, 55455 Minneapolis, MN, USA a large number of elderly individuals are cognitively impaired. WHO estimates that at the moment 47.6 million people have dementia, a disease that challenges the affected individuals, their relatives and society [1]. It is still unclear to what extent general cognitive decline in elderly individuals is caused by normal aging processes or diseases, but in fact mental health varies immensely between individuals in their late life, thus studying mental health among elderly individuals is highly relevant. A substantial fraction of those who live to be 100 years old remain mentally healthy, exemplifying that some elderly individuals achieve successful cognitive ageing [2].

In recent times, epigenetic signatures have been exploited to address the underlying causes of neurodegeneration, and microRNAs (miRNAs) in the blood have been introduced as novel biomarkers of neurodegenerative disease [3], such as dementia and in particular Alzheimer Disease (AD), the most common form of dementia. MiRNAs are of particular interest as they regulate protein expression posttranscriptionally usually by binding complementary sites of the $3^{\prime}$ untranslated region (UTRs) of their target messengerRNAs, thus introducing cleavage or inhibition of translation 
[4, 5]. MiRNAs are 19-22 nucleotide (nt) non-coding RNAs that are usually formed as cleaved products from larger stem loop folded precursor RNAs of usually $61 \mathrm{nt}$, thus several miRNAs originate from the same precursor. MiRNA can be released from a variety of cell types to body fluid circulation, e.g., from brain neurons to the extracellular environment, by binding to RNA-binding proteins or by transportation in exosomes and micro-vesicles [6]. MiRNAs are remarkably stable, and readily detectable, in plasma and serum, even after e.g., freezing and thawing samples repeatedly [7].

MiRNA dysregulation in AD patients has been studied both through applied candidate approaches and using larger panels of miRNAs in discovery studies. A recent review summarizes results from 18 studies of miRNA and $\mathrm{AD}$ or cognitive impairment adding to a total of 2600 participants. From this study, there is no consensus regarding which biological material should be used, some use plasma/serum, others peripheral blood mononuclear cells or cerebrospinal fluid (CSF). Also, it is stressed that there are population differences and other factors, such as lifestyle, varying between studies that could affect miRNA expression. The most consistent finding is that miR-132 is upregulated in serum/plasma in AD patients compared with controls in three independent studies while a handful of miRNAs did replicate in at least two studies [8].

In addition to $\mathrm{AD}$, changes in miRNA expression have been associated with Mild Cognitive Impairment (MCI) among elderly individuals. In a candidate study by Sheinerman et al. miR-132 and miR-134 were upregulated in plasma from participants with MCI when compared to age matched controls [9], while Tan et al. showed that miR$125 \mathrm{~b}$ was correlated with mini mental state examination scores (MMSE) in AD patients [10]. MiR-132 and miR-206 was also studied by Xie et al. who found both miRNAs upregulated in serum of persons with MCI [11]. Most studies are cross-sectional or case-control based while longitudinal studies are rare, in one such follow-up study of individuals with amnestic MCI miR-206 in serum was found to be an even better predictor for AD than miR-132 [12]. A certain degree of overlap seems to exist between miRNA's associated with both $\mathrm{AD}$ and MCI, and thus it is reasonable to hypothesize that at least some miRNA candidates of $\mathrm{AD}$ also contribute to normal aging neurobiology.

We aim to pursue the rationale of shared miRNA biology between dementia and normal cognitive variation in twins 73-95 years of age. Also, we aim to deduce the general trend of associations between circulating miRNAs and both the level and decline of cognitive scores over a period of up to 10 years. Finally, we will keep the options open for discovering novel miRNAs associated with normal aging neurobiology using both paired (twin-pair level) and ordinary individual-level test statistics.

\section{Material and methods}

\section{Study population}

The study participants were selected from the Longitudinal Study of Aging Danish Twins (LSADT), which is a longitudinal study of Danish twins aged 70 years and older [13, 14]. The study was initiated in 1995 , and the survey was repeated in 1997, 1999, 2001, 2003, 2005 and 2007, although no cognitive tests were performed in 2007. In 1997, mainly twin pairs of the same sex who were 73 years of age or more were included in the present study. Whole blood samples were drawn in 1997 from 689 members of twin pairs into $\mathrm{K}_{2}$-EDTA tubes (BD Bioscience). Within $1 \mathrm{~h}$ after blood collection, plasma was prepared by centrifuging the tubes $2000 \times g$ for $10 \mathrm{~min}$ at $4^{\circ} \mathrm{C}$. Plasma was transferred to RNase free tubes and stored at $-80{ }^{\circ} \mathrm{C}$.

The present study included 45 monozygotic twin pairs who were randomly selected from the twin pairs, where both individuals donated plasma and were classified as monozygotic twins using questionnaire-based zygosity assessment [15].

All methods were carried out in accordance with relevant guidelines and regulations. Written informed consents were obtained from all participants, and all surveys and use of survey information were approved by The Regional Committees on Health Research Ethics for Southern Denmark (VF 20040241).

Data access: according to Danish legislation, transfer and sharing of individual-level data requires prior approval from the Danish Data Protection Agency and requires that the data sharing requests be dealt with on a case-by-case basis. The data have been deposited to the European Genomephenome Archive (https://www.ebi.ac.uk/ega/home) with accession number EGAS00001002887.

\section{Cognitive function}

Cognitive functioning was assessed using the MMSE and a five-component CCS [16]. The widely used MMSE ranges from 0 to 30 and can be graded as severely impaired for scores between 0 and 17, mildly impaired for scores between 18 and 23, and non-impaired for scores between 24 and 30. The CCS is an in-house developed combination of cognitive tests that has been used in several middle-aged and elderly cohorts. The CCS items were originally selected to represent tasks that are sensitive to normative age changes, which could additionally be reliably and briefly assessed by lay interviewers. The specific tasks 
included a fluency test, which involved the number of animals an individual could name in a 1-min interval, forward and backward digit span, and immediate and delayed recall of a 12-item list. The cognitive composite score was computed by taking the sum of the five standardized measures.

\section{RNA extraction and plasma miRNA profiling}

Total RNA including small RNA was extracted from $100 \mu \mathrm{l}$ of plasma using the mirVana PARIS kit (Life Technologies) according to the manufacturer's instructions for liquid samples. RNA was eluted with $100 \mu \mathrm{l}$ of RNase free water.

The expression of 754 miRNAs in plasma was measured by semi-quantitative real-time PCR (qRT-PCR) using the TaqMan OpenArray Human microRNA panel (Life Technologies). Total RNA was converted to cDNA using the TaqMan MicroRNA Reverse Transcription Kit and Megaplex stem-loop RT primers for Human Pool A and B (Life Technologies) according to the manufacturer's instructions for low sample input (LSI). For each RNA sample reverse transcription (RT) was performed in two separate reactions; one with Pool A and one with Pool B Megaplex RT primers. Each pooled TR reaction had a final volume of $7.5 \mu \mathrm{l}$ and contained $3 \mu$ l of total RNA. Pool A and Pool B Megaplex RT primer sets each allow for simultaneous cDNA synthesis of 377 unique miRNAs.

Pre-amplification was performed using Megaplex PreAmp primers for Human Pool A or Pool B (Life Technologies) and TaqMan PreAmp Mastermix (Life Technologies) according to the manufacturer's instructions for LSI. PreAmp reactions had a final volume of $40 \mu \mathrm{l}$ and contained $7.5 \mu \mathrm{l}$ RT-product. Thermal cycling conditions were as follows: $95^{\circ} \mathrm{C}$ for $10 \mathrm{~min}, 55^{\circ} \mathrm{C}$ for $2 \mathrm{~min}$, and $72^{\circ} \mathrm{C}$ for 2 min, followed by 16 cycles of $95^{\circ} \mathrm{C}$ for $15 \mathrm{~s}$ and $60^{\circ} \mathrm{C}$ for $4 \mathrm{~min}$. Final inactivation was performed at $99.9^{\circ} \mathrm{C}$ for 10 min. PreAmp products were diluted 1:40 in $0.1 \times$ TE buffer (pH 8.0).

For the qRT-PCR step $22.5 \mu \mathrm{l}$ of diluted PreAmp product was mixed with $22.5 \mu \mathrm{l}$ TaqMan OpenArray Real time Master Mix (Life Technologies) and loaded into each of eight wells ( $5 \mu \mathrm{l}$ in each well) on an OpenArray 384-well sample loading plate to obtain an usable format for automatic pipetting. TaqMan OpenArray Human microRNA panels were then automatically loaded using the OpenArray AccuFill System (Life Technologies). Loaded OpenArray panels were cycled in an OpenArray NT Cycler System (Life Technologies) using the OpenArray Real-Time qPCR Analysis Software (v1.0.4) with a pre-assigned cycling program. Each OpenArray panel enables the quantification of 754 human miRNAs in three samples. Samples from a twin-pair were quantified on the same panel with a notemplate control (NTCs) or samples from three twin pairs were quantified simultaneously on two panels. In total, two panels included NTCs.

\section{miRNA quantification and normalization}

The OpenArray Real-Time qPCR Analysis Software was used to obtain quantification cycle $(\mathrm{Cq})$-values, which were exported to the $\mathrm{qBase}{ }^{\mathrm{PLUS}}$ software (Biogazelle, NV, Belgium) for relative quantification. Cq-values were converted to relative quantities using the method of $\mathrm{qBas} \mathrm{e}^{\mathrm{PLUS}}$, which is a modification of the classic delta-delta-Cq method that includes multiple reference gene normalization [17].

Normalization was performed by dividing the relative quantities by a sample specific normalization factor, which is calculated by taking the geometric mean of the relative quantities of selected reference genes [17]. Assessment of putative reference genes for normalization was evaluated using geNorm [18] as an implementation of the $\mathrm{qBase}^{\text {PLUS }}$ software. Pool A and Pool B miRNA data were normalized separately. MiRNAs with data for all 90 samples were considered as candidates for normalization and therefore evaluated using geNorm. Reference genes were selected based on the average expression stability value $M$ and the average pairwise variation $V$ of a particular gene with all other tested reference genes. Also, to avoid selection of coregulated miRNAs, only one miRNA per miRNA family was selected for normalization. On the basis of this, seven miRNAs (hsa-miR-335-5p, hsa-miR-26a-5p, hsa-miR-1915p, hsa-miR-328-3p, hsa-miR-106b-5p, hsa-miR-26b-5p and hsa-miR-20a-5p) were selected as reference genes for pool A miRNA data, while one miRNA (hsa-miR-126-5p*) and one small RNA (U6 rRNA) were selected as reference genes for pool B miRNA data. For downstream analysis, only miRNA expressed in at least 10 samples were included and for the paired analysis at least five pairs of twins should have a given miRNA expressed in plasma from both twinpair-members.

\section{Twin-pair level analyses}

The co-twin design was used to detect the association of miRNAs with CCS and MMSE using a hypothesis-free approach. The twin design is advantageous by controlling the genetic influence on the phenotype variation because the genetic effects are canceled out in the twin-pair level analysis of genetically identical MZ twins, also shared family environment is controlled for by the design [19]. The analysis fits a linear regression model that regresses the intrapair difference in miRNA expression on the intra-pair difference in CCS or MMSE, with intra-pair differences calculated between the twin with the higher phenotype value to the twin with the lower phenotype value, i.e., we fitted $\Delta$ miRNA $=\beta_{0}+\beta_{1} \times \Delta$ phenotype $+\beta_{2} \times$ age $+\beta_{3} \times$ sex. In 
Table 1 Demographics of cohort participants from the Longitudinal study of Aging Danish Twins (LSADT)

\begin{tabular}{ll}
\hline & LSADT \\
\hline No. MZ-twins & 90 \\
No. twin-pairs & 45 \\
No. female pairs, (\%) & $34(78)$ \\
Assessment year 1997: & \\
Age (sd) & $78.7(3.5)$ \\
No. MMSE obs. & 90 \\
No. CCS obs. & 90 \\
MMSE (sd) & $26.37(2.57)$ \\
CCS (sd) & $1.06(2.91)$ \\
$\Delta$ MMSE (sd) & a \\
$\Delta$ CCS (sd) & $2.17(1.45)$ \\
Cognitive growth curve: & $2.88(2.40)$ \\
No. MMSE assessments & \\
MMSE mean level (sd) & 337 \\
MMSE slope (sd) & \\
No. CCS assessments & $26.63(0.26)$ \\
CCS mean level (sd) & $-0.23(0.06)$ \\
CCS slope (sd) & 327 \\
\hline
\end{tabular}

${ }^{a}$ Quantitative differences (Absolute difference) between the cognitive performance of the twin with the best cognitive score subtracted by the performance of the co-twin with-in a pair

${ }^{\text {b }}$ Yearly decline. $p$ value $<0.001$

${ }^{c}$ Yearly decline. $p$ value $=0.002$

this model, $\beta_{0}$ captures the overall difference in miRNA level between higher and lower phenotype twins when age was centered around its median and the phenotype differences was also centered at the median of the absolute differences (difference in mean), while $\beta_{1}$ stands for the quantitative association of miRNA that is dependent on the level of phenotype difference (degree of discordance). When miRNA expression is log transformed, the antilog of $\beta_{0}$ is the fold change in miRNA expression between higher and lower phenotype twins and the antilog of $\beta_{1}$ is the fold change in miRNA expression for one unit increase in intrapair phenotype difference. The effects of age and sex (women coded as 0 and men coded as 1) on intra-pair difference in miRNA expression were adjusted by including them as pair-specific variables in the regression model. The intra-pair analysis was performed using linear regression with the $\operatorname{lm}()$ function in the statistical computational environment R (https://www.r-project.org).

\section{Individual level analysis}

In addition to intra-pair analysis, we also performed individual level cross-section analysis by defining twin pairing as clusters in a linear mixed model with random intercept.
The fitting was done with the lmer() function from the Rpackage lme4 [20]. For low abundant miRNAs expressed only in one twin-pair or only in one of the twins in the pairs, we fitted a simple linear regression model. The models detect associations between candidate miRNA and the mean cognitive level by regressing miRNA expression on cognitive performance. Similar to the intra-pair analysis, models were adjusted for age and sex by including them as co-variates.

In both twin-pair and individual analyses, age was centered by subtracting the median age of 78.1 from the observed individual ages. Cognitive functioning was analyzed as a continuous variable using either the CCS or the MMSE scores. Although in the individual and twin-pair analyses we correct for multiple testing using an FDR approach before declaring a significant result, consistent with the exploratory nature of this study we report all uncorrected $p$ values $<0.05$.

\section{Level of cognitive scores and cognitive decline}

Candidate miRNAs with $p<0.05$ from the individual crosssectional analysis (30 miRNAs) were subsequently included in a post-hoc statistical longitudinal analysis. We used a random effects/growth curve model approach to perform analyses of associations primarily with the slope of cognitive functioning (reflecting change in cognitive functioning over time) but also the mean cognitive level (i.e. the intercept). The intercept (regression coefficient, level) was defined as the level of the cognitive functioning at the mean age of 78.7 when the blood was donated while the slope (regression coefficient, slope) is defined as the age-related decline as previously described [21]. Cognitive functioning was analyzed as a continuous variable using either the CCS or the MMSE score, adjusted for age and sex. Corrections for multiple testing were not applied to these analyses of allele effects, since an a priori hypothesis of association was assumed for each of the 30 miRNA candidates. As the main purpose was to study the association with cognitive decline, we undertook a one-tailed test with the tail specified by the direction in the cross-sectional analyse in Table 3, assuming a lower cognitive level also associate with a steeper cognitive decline. Results from the random effects model that passes a 5\% level of significance were marked in italic fonts, further those also passing a 5\% level of significance on slope of cognitive functioning were additionally marked in bold. The random effects models were fitted using the mixed model function in STATA 14.2 (StataCorp, Texas, USA), as previously described [21]. Power analysis was performed with the R-package sizepower (Bioconductor) allowing for one false positive. With the 211 miRNA's large sample size, a power of $77 \%$ was provided to detect expression differences of 1.2 -fold for miRNA with no 
Table 2 miRNA associated with cognitive function using the matched-pair twin design

\begin{tabular}{|c|c|c|c|c|c|}
\hline \multirow[t]{2}{*}{ miRNA } & \multirow[t]{2}{*}{$\begin{array}{l}\text { No. twin } \\
\text { pairs }^{\text {a }}\end{array}$} & \multicolumn{2}{|c|}{$\begin{array}{l}\text { Difference in } \\
\text { mean }\end{array}$} & \multicolumn{2}{|c|}{$\begin{array}{l}\text { Degree of } \\
\text { discordance }\end{array}$} \\
\hline & & $\beta_{0}$ & $p$ value & $\beta_{1}$ & $p$ value \\
\hline \multicolumn{6}{|l|}{ CCS: } \\
\hline hsa-miR-20b-5p & 43 & -0.07 & 0.44 & 0.10 & 0.002 \\
\hline hsa-miR-223-5p & 32 & 0.09 & 0.29 & -0.12 & 0.004 \\
\hline hsa-miR-15b-5p & 44 & -0.03 & 0.50 & -0.05 & 0.004 \\
\hline hsa-miR-132-3p & 41 & 0.14 & 0.13 & -0.09 & 0.005 \\
\hline hsa-miR-483-5p & 45 & -0.06 & 0.56 & 0.11 & 0.008 \\
\hline hsa-miR-766-3p & 44 & -0.16 & 0.12 & -0.09 & 0.01 \\
\hline hsa-miR-543 & 13 & -0.26 & 0.05 & -0.17 & 0.01 \\
\hline hsa-miR-9-3p & 23 & -0.20 & 0.15 & -0.15 & 0.02 \\
\hline hsa-miR-155-5 $\mathrm{p}^{\mathrm{b}}$ & 36 & -0.08 & 0.47 & 0.09 & 0.02 \\
\hline hsa-miR-212-3p & 14 & 0.11 & 0.31 & 0.14 & 0.02 \\
\hline hsa-miR-320b & 37 & -0.01 & 0.97 & -0.13 & 0.02 \\
\hline hsa-miR-151a-3p & 36 & 0.03 & 0.85 & -0.14 & 0.02 \\
\hline hsa-miR-146b-3p & 44 & -0.03 & 0.39 & 0.03 & 0.03 \\
\hline hsa-miR-383-5p & 23 & -0.23 & 0.02 & 0.08 & 0.03 \\
\hline hsa-miR-1274b & 44 & -0.07 & 0.33 & -0.05 & 0.04 \\
\hline hsa-miR-27a-3p & 40 & 0.13 & 0.03 & -0.04 & 0.05 \\
\hline \multicolumn{6}{|l|}{ MMSE: } \\
\hline hsa-miR-548c-3p & 44 & 0.31 & 0.05 & 0.26 & 0.004 \\
\hline hsa-miR-539-5p & 12 & 0.05 & 0.72 & 0.18 & 0.04 \\
\hline hsa-miR-532-3p & 30 & 0.14 & 0.27 & -0.15 & 0.04 \\
\hline hsa-miR-369-3p & 11 & -0.01 & 0.97 & 0.17 & 0.04 \\
\hline mmu-miR-134-5p & 40 & -0.02 & 0.80 & 0.11 & 0.04 \\
\hline hsa-miR-548a-3p & 44 & 0.01 & 0.92 & 0.15 & 0.04 \\
\hline hsa-miR-27a-5p & 5 & -2.05 & 0.05 & -1.50 & 0.05 \\
\hline
\end{tabular}

MiRNA associated with Mini Mental State Examination (MMSE) or Cognitive Composite Score (CCS) with a significance of lower than the $5 \%$ level is presented either by the difference in mean at centered co-variates or the degree of discordance. All analyses were adjusted for age and sex between pairs

${ }^{a}$ Number twin pairs where both twins expressed the miRNA

${ }^{\mathrm{b}}$ Significant by age $p<0.05$

missing readings $\left(N_{\text {pairs }}=45\right)$ and a comparable power of $80 \%$ to detect expression differences of 1.4-fold for miRNAs with missing readings $\left(N_{\text {pairs }}=14\right.$, e.g.,s for miR-212-3p).

\section{Results}

\section{Circulating miRNA and cognitive function}

Of the 754 miRNAs incorporated on the OpenArray platform, a total of 278 miRNAs were expressed in plasma from at least 10 of the 90 study participants. We first pursued to discover plasma miRNAs associated with cognitive scores in the same year the blood was donated using paired and individual analysis. Cognitive scores, displayed in Table 1, indicate that the twins were mainly cognitive healthy when blood was donated. None were severely cognitively impaired (MMSE < 17) but $14(16 \%)$ were mildly impaired (MMSE < 24). The absolute twin-pair discordance between the best and the worse performing twin when the blood was donated was on average less than three units apart on both the MMSE and CCS scales equivalent to approximately one standard deviation of the individual variation on each of the scales. In addition, using the repeated assessments that averaged to 3.7 (MMSE) and 3.6 (CCS) assessments per participant we saw as expected mean levels similar to in the growth curve model as those estimated cross-sectional (year 1997) and a yearly decline in both cognitive scores (Table 1). MMSE and CCS were positively correlated (reg. coef. $0.44, p$ value $<0.001$ ), but there was also a large degree of independent variation $\left(R^{2}=0.15\right)$.

\section{Twin-pair level difference in cognitive scores and miRNA}

For the matched-pair twin analysis, a total of 211 miRNAs passed our minimum abundance criterion, which is at least that five pairs of twins should have a given miRNA expressed in plasma from both twin-pair-members. A total of 16 miRNAs were associated with difference in CCS, while seven miRNAs were associated with MMSE ( $p$ values $<0.05$ ), but none of these associations remained significant when adjusted for multiple testing by False Discovery Rate (FDR), thus they are referred to as nominally significant. In Table 2 miR-543 (CCS) and miR-27a-5p (MMSE) are examples of inverse association at the difference in means $\left(\beta_{0}\right)$, which is the mean difference of miRNA expression between the highest cognitive performing twin and the lowest cognitive performing co-twin, when all covariates are centered. But also at the degree of discordance $\left(\beta_{1}\right)$, which is the regression coefficient of the intra-pair miRNA difference and intra-pair cognitive difference e.g., for twins with good cognitive function, miRNAs were down regulated compared with the co-twin and other twins who performed poorer on the cognitive scale. MiR-548c-3p (MMSE) is an example of positive association on both levels, but there was no general consistency of association on the two levels.

None of the miRNAs were associated with both cognitive measures, i.e., CCS and MMSE, yet miR-27a-3p, was inversely associated with CCS, while miR-27a-5p was associated inversely with MMSE, which may be considered a supportive finding since both miRNAs derive from premiR-27 (Table 2). 


\section{Individual-level association between cognitive scores and miRNA}

An ordinary individual cross-sectional analysis was also performed, which include analysis of low abundant miRNAs. In the individual analysis, 24 miRNAs were significantly associated with CCS and six were associated with MMSE (Table 3), but again none of the associations remained significant when adjusted for multiple testing (FDR). Of these 30 miRNAs, 24 were inversely associated, while six were positively associated with the cognitive measures. Of note, ten of the miRNAs were low-abundance miRNAs, i.e., observed in less than $1 / 3$ of the samples (Table 3). Among the 20 high-abundant miRNA hits, three miRNAs were particularly interesting as they were associated with CCS in both the paired and individual-level analysis. MiR-151a-3p and miR-1274b were inversely associated with CCS, while miR-212-3p was positively associated with CCS as displayed in Fig. 1.

\section{Level of cognitive scores and cognitive decline}

As the twins were assessed biennially, we made use of this information by applying a growth curve model in a longitudinal analysis of the 30 miRNAs initially associated with the cognitive scores on the individual level shown above (Table 3). For four miRNAs, miR-151a-3p, miR-18a-5p, miR-134-5p and miR-320a a higher miRNA expression was associated with a steeper slope (one sides test, $p$ values < $0.05)$, i.e., associated with an increased age-related cognitive decline, indicating potential as early biomarkers for age-related decline in cognitive performance. Of note, higher miR-1274b expression was associated with less steep slope of decline (two-sided $p$ value $=0.04)($ Table 4).

The consistency of association with cognitive level between models used in Tables 3 and 4 was also investigated and here we confirmed associations in the same direction between 10 miRNAs and CCS, and three miRNAs were associated with MMSE (one-sided test, $p$ values < 0.05) when centered at the age 78.7. All miRNAs except two were inversely associated with cognitive scores.

\section{Discussion}

In the present work, we explored the association of circulating miRNAs in plasma in relation to cognitive function among generally healthy elderly twins, and revealed multiple miRNAs that tend to be associated with cognitive function although we found no major significant association. The list of 23 nominally significant miRNAs identified using the paired test statistics included both novel findings and miRNAs previously found to have a role in
Table 3 miRNA expression associated with cognitive function, Mini Mental State Examination (MMSE) or Cognitive Composite Score (CCS) on the individual level

\begin{tabular}{|c|c|c|c|}
\hline miRNA & No. individuals & $\begin{array}{l}\text { Regression } \\
\text { coefficient }\end{array}$ & $p$ value \\
\hline \multicolumn{4}{|l|}{ CCS: } \\
\hline hsa-miR-206 & 23 & -0.22 & 0.001 \\
\hline hsa-miR-497-5p & 42 & -0.09 & 0.003 \\
\hline hsa-miR-769-5 $\mathrm{p}^{\mathrm{a}}$ & 13 & -0.21 & 0.003 \\
\hline hsa-miR-151a-3p & 80 & -0.08 & 0.004 \\
\hline hsa-miR-1249-3p ${ }^{a}$ & 16 & -0.16 & 0.004 \\
\hline hsa-miR-1274a & 88 & -0.05 & 0.005 \\
\hline hsa-miR-520e & 36 & 0.20 & 0.01 \\
\hline hsa-miR-18a-5p & 60 & -0.04 & 0.01 \\
\hline mmu-miR-134-5p & 84 & -0.05 & 0.01 \\
\hline hsa-miR-190b ${ }^{a}$ & 15 & -0.21 & 0.01 \\
\hline hsa-miR-320a & 89 & -0.02 & 0.02 \\
\hline hsa-miR-1227-3p & 28 & -0.08 & 0.02 \\
\hline hsa-miR-340-3p & 86 & -0.03 & 0.02 \\
\hline hsa-miR-720 & 89 & -0.04 & 0.03 \\
\hline hsa-miR-30a-3p & 31 & 0.06 & 0.03 \\
\hline hsa-miR-425-3p & 48 & -0.06 & 0.03 \\
\hline hsa-miR-212-3p & 49 & 0.05 & 0.03 \\
\hline hsa-miR-145-5p & 47 & -0.04 & 0.03 \\
\hline hsa-miR-1274b & 89 & -0.02 & 0.04 \\
\hline hsa-miR-516-3p & 20 & 0.06 & 0.04 \\
\hline hsa-miR-452-5p & 22 & 0.07 & 0.04 \\
\hline hsa-miR-625-3p & 79 & -0.05 & 0.05 \\
\hline hsa-miR-130b-5 $\mathrm{p}^{\mathrm{a}}$ & 11 & -0.08 & 0.05 \\
\hline hsa-miR-410-3p & 83 & -0.03 & 0.05 \\
\hline \multicolumn{4}{|l|}{ MMSE: } \\
\hline hsa-let-7g-5p & 83 & -0.04 & 0.003 \\
\hline hsa-miR-638 & 51 & 0.08 & 0.01 \\
\hline hsa-miR-15a & 64 & -0.04 & 0.02 \\
\hline hsa-miR-505-3p & 38 & -0.04 & 0.03 \\
\hline hsa-miR-505-5 $\mathrm{p}^{\mathrm{a}}$ & 10 & -0.06 & 0.04 \\
\hline hsa-miR-664a-3p & 21 & -0.10 & 0.05 \\
\hline
\end{tabular}

Significant association $(p$ value $<0.05)$ adjusted for age, sex and family relation (twin pairing) are displayed in the table

${ }^{a}$ Less than two-complete pairs and the $1 \mathrm{~m}$ model was used

neurodegeneration or neuro-pathology. Interestingly, three miRNAs miR-151a-3p, miR-212-3p and miR-1274b were associated with CCS in both the individual and the paired analysis. We further substantiated some of these findings using repeated assessment data and thereby confirmed thirteen of the associations from the individual analysis. Overall, the analyses indicated that elderly individuals with poor cognitive function had upregulated plasma miRNAs compared with equivalent individuals who performed better on the cognitive scale. Also, upregulated miRNAs 

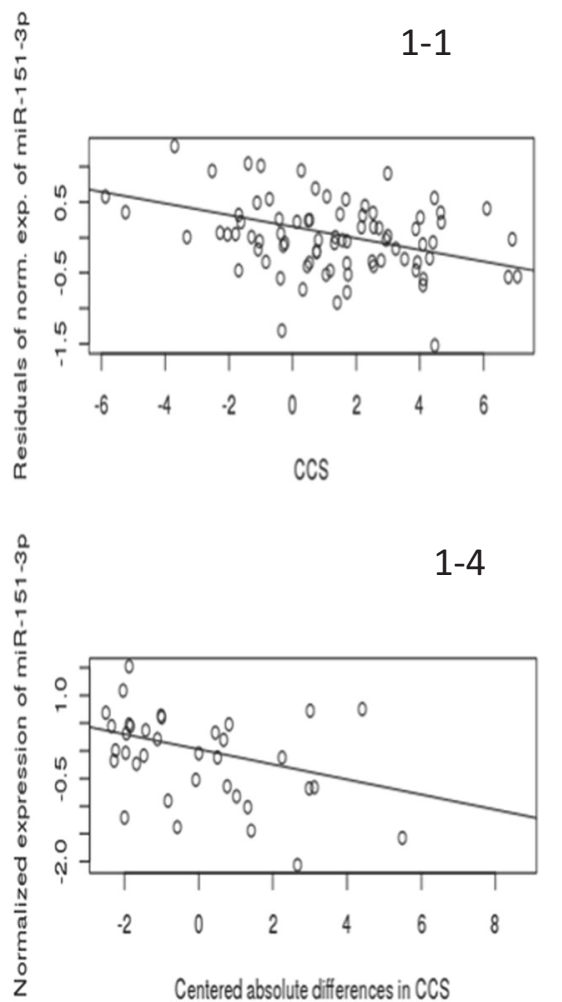
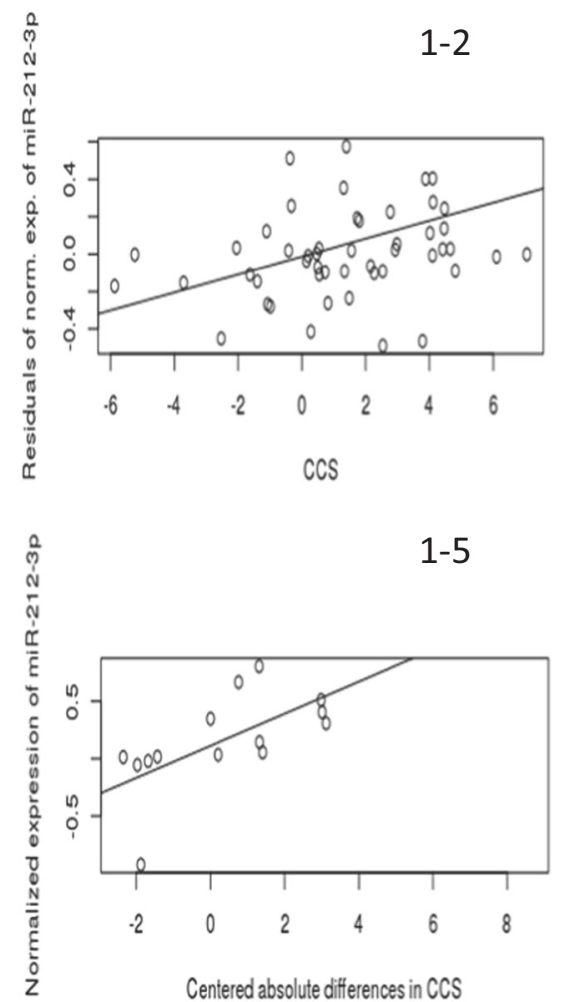

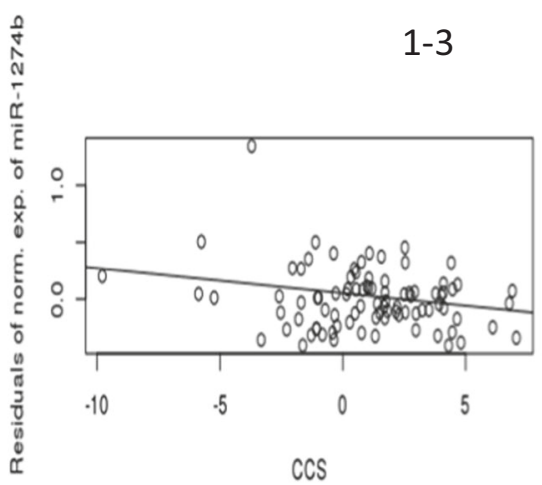

$1-6$

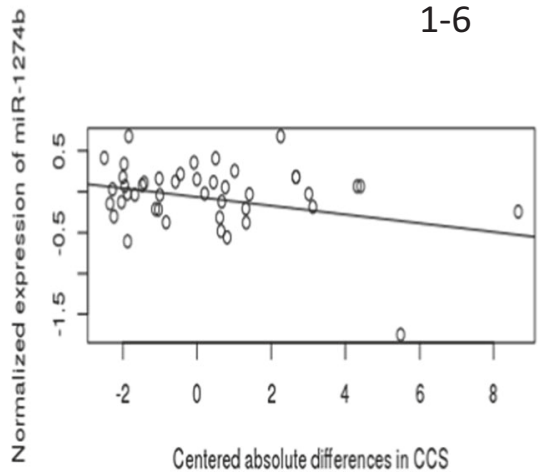

Fig. 1 Plots of three miRNAs miR-151-3p, miR212-3p and miR$1274 \mathrm{~b}$ associated with cognitive composite score (CCS) from individual and paired analysis. Individual analysis: 1-1 Scatter plot of cognitive composite score (CCS) and residuals from the linear model of the expression of miR-151-3p with the line of regression. 1-2 Scatter plot of CCS and residuals from the linear model of the expression of miR-212-3p with the line of regression. 1-3 Scatter plot of CCS and residuals from the linear model of the expression of

associated with steeper longitudinal decline in CCS for four miRNAs.

The list of nominally significant findings included miRNAs previously associated with $\mathrm{AD}$ or MCI biomarkers thus suggesting a partly shared biology between $\mathrm{AD}$, MCI and normal cognitive aging. One such miRNA, miR-206, has previously been reported as being associated with $\mathrm{AD}$ and among individuals with amnestic MCI it was a better predictor for AD than BDNF, which is a target of miR-206 [12]. MiR-206 is a member of the miR-1 gene family, which includes miR-1-1, miR-1-2, miR-206, miR-133a-1, miR-133a-2, and miR-133b, but in the present work, miR206 was the only family member among the nominally significant results. MiR-206 is potentially a participant in the biological mechanisms of learning or memory, nerve development, neuron recognition, and in the regulation of neuron differentiation, which are closely related to the development of $\mathrm{AD}$ [22]. Interestingly, in an animal experiment, synthetic miR-206 inhibitor has even shown to increase the brain levels of BDNF and to enhance the hippocampal synaptic density as well as neurogenesis [22]. These data thus suggest that the regulation of BDNF could
miR-1274b-3p with the line of regression. Twin-paired analysis: 1-4 Scatter plot of centered absolute difference in CCS and normalized expression of miR-151-3p with the line of regression. 1-5 Scatter plot of centered absolute difference in CCS and normalized expression of miR-212-3p with the line of regression. 1-6 Scatter plot of centered absolute difference in CCS and normalized expression of miR-1274b with the line of regression

be part of the determinants of cognitive function in the normal range of mainly healthy elderly individuals.

Another nominally significant miRNA known to have a central role in the pathology of $\mathrm{AD}$ is miR-27a-3p which e.g., Frigerio et al. reported to be significantly reduced in $\mathrm{CSF}$ of $\mathrm{AD}$ compared to controls and to correlate with the levels of the AD biomarkers tau, p-tau and A $\beta 1-42$ [23]. Although Denk et al. could not replicate these findings in a later study [24], we observed the same miRNA to be associated with CCS (Table 2). Another indication that beta-amyloid plaque formation in the brain could be part of the biology shared between $\mathrm{AD}$ and normal cognitive aging comes from miR-9, which we found to be associated with CCS in the paired analysis (Table 2). MiR-9 is enriched in the brain [25] and is suggested to be part of a network that indirectly regulates the APP processing, $\mathrm{A} \beta$ production and accumulation [26]. The list of miRNAs that we found to be associated with cognitive scores also included one of the most prominent miRNAs in AD, miR-132, which was found to be upregulated in $\mathrm{AD}$ patients in body fluids in three independent studies [8] and which, together with miR-212, is dysregulated in post-mortem brains [27]. Both 
Table 4 Selected miRNA candidates (30 miRNA, from Table 3) associated with cognitive function and cognitive decline in Mini Mental State Examination (MMSE) or Cognitive Composite Score (CCS) using growth curve models. All analyses were adjusted for age and sex

\begin{tabular}{|c|c|c|c|c|c|}
\hline \multirow[b]{2}{*}{ miRNA } & \multirow[b]{2}{*}{ No. of assessments (No. of individuals) } & \multicolumn{2}{|l|}{ Level } & \multicolumn{2}{|l|}{ Slope } \\
\hline & & Regression coefficient & $p$ value & Regression coefficient & $p$ value \\
\hline \multicolumn{6}{|l|}{ CCS: } \\
\hline hsa-miR-206 & $82(23)$ & -1.00 & 0.02 & -0.03 & 0.47 \\
\hline hsa-miR-497-5p & $148(42)$ & -0.46 & 0.26 & 0.12 & 0.85 \\
\hline hsa-miR-769-5p & $46(13)$ & -0.41 & 0.25 & 0.01 & 0.51 \\
\hline hsa-miR-151a-3p & $294(80)$ & -0.78 & 0.04 & -0.13 & 0.05 \\
\hline hsa-miR-1249-3p & $63(16)$ & -1.32 & 0.07 & -0.07 & 0.38 \\
\hline hsa-miR-1274a & $322(88)$ & -1.11 & 0.03 & 0.06 & 0.69 \\
\hline hsa-miR-520e & $135(36)$ & 0.01 & 0.49 & -0.06 & 0.94 \\
\hline hsa-miR-18a & $210(60)$ & -2.45 & 0.002 & -0.39 & 0.04 \\
\hline$m m u-m i R-134$ & $304(84)$ & -0.91 & 0.03 & -0.20 & 0.04 \\
\hline hsa-miR-190b & $56(15)$ & -2.27 & 0.002 & 0.10 & 0.74 \\
\hline hsa-miR-320a & $324(89)$ & -1.97 & 0.02 & -0.57 & 0.005 \\
\hline hsa-miR-1227-3p & $98(28)$ & -1.34 & 0.03 & -0.23 & 0.19 \\
\hline hsa-miR-340-3p & $314(86)$ & -0.76 & 0.19 & -0.33 & 0.02 \\
\hline hsa-miR-720 & $321(89)$ & -0.92 & 0.06 & -0.01 & 0.48 \\
\hline$h s a-m i R-30 a-3 p$ & $112(31)$ & 2.00 & 0.02 & 0.16 & 0.13 \\
\hline hsa-miR-425-3p & $186(48)$ & -0.34 & 0.31 & 0.03 & 0.62 \\
\hline hsa-let-212-3p & $190(49)$ & -0.44 & 0.73 & -0.01 & 0.47 \\
\hline hsa-miR-145-5p & $185(47)$ & -0.05 & 0.48 & -0.11 & 0.26 \\
\hline hsa-miR-1274b & $321(89)$ & -1.50 & 0.05 & 0.36 & $0.98^{b}$ \\
\hline hsa-miR-516-3p & $75(20)$ & -1.34 & 0.93 & -0.31 & 0.17 \\
\hline hsa-miR-452-5p & $83(22)$ & 1.17 & 0.17 & 0.11 & 0.33 \\
\hline hsa-miR-625-3p & $283(79)$ & -0.56 & 0.10 & -0.09 & 0.19 \\
\hline \multicolumn{6}{|l|}{ hsa-miR-130b-5p $\mathrm{p}^{\mathrm{a}}$} \\
\hline hsa-miR-410-3p & $296(83)$ & -0.75 & 0.11 & -0.24 & 0.05 \\
\hline \multicolumn{6}{|l|}{ MMSE: } \\
\hline hsa-let- $7 g-5 p$ & $314(83)$ & -1.48 & 0.05 & -0.02 & 0.47 \\
\hline hsa-miR-638 & $192(51)$ & 0.83 & 0.05 & -0.11 & 0.81 \\
\hline hsa-miR-15a & $239(64)$ & -1.02 & 0.08 & 0.06 & 0.61 \\
\hline hsa-miR-505-3p & $36(10)$ & -16.44 & 0.002 & -0.18 & 0.43 \\
\hline hsa-miR-505-5p & $148(38)$ & 0.05 & 0.48 & 0.20 & 0.16 \\
\hline hsa-miR-664a-3p & $83(21)$ & -0.05 & 0.48 & -0.15 & 0.11 \\
\hline
\end{tabular}

Results in italic fonts had $p$ values $<0.05$ for a one-sided test on the cognitive level, results in bold also had one-sided test $p$ values $<0.05$ on the slope (cognitive decline)

${ }^{\mathrm{a}}$ Model did not complete analysis

${ }^{\mathrm{b}} p$ value $=0.04$ then using a two-sided test

miR-132 and miR-212 co-localize in a complex that is involved in neuronal migration, axonal growth, and neuronal morphology $[28,29]$ and have been linked to several pathways playing a major role in the pathogenesis of $\mathrm{AD}$ [27]. MiR-212-3p was also positively associated with CCS in the present work, both in the individual and the paired analyses, whereas miR-132-3p was inversely associated with CCS in the paired analyses (Table 2 and 3). The upregulation of miR-132-3p among cognitively poorly performing individuals thus support previous findings from AD studies $[8,9,11]$. These observations are also in line with other studies that have uncovered a shared biology between AD and MCI [9-12], and further supported by the observed pleiotropy of AD risk alleles with normal cognitive aging in our previous genetic studies [21, 30, 31].

In addition to the previously known miRNAs, we identified miRNAs that could play a role in normal cognitive aging, but where knowledge about their regulation in 
neurobiology is sparse. Such candidates are miR-1274b and miR-151a-3p that we found associated with CCS in both the individual and the paired analyses. To our knowledge, there is limited information of the possible involvement of miR$1274 \mathrm{~b}$ in cognitive function, and thus this could be a novel finding, although it has been questioned if the putative mature miR-1274 sequence is in fact a fragment of a Lys tRNA [32]. However, it is plausible that miR-151a-3p plays a role in cognition as it is expressed in neurons from animals and associate with e.g., Parkinson's disease and AD in human studies [8, 33, 34]. Noteworthy, miR-151a-3p also associated with age-related cognitive decline. Another miRNA, miR-505-5p, which we found nominally associated with cognitive function is worth mentioning, since Denk et al. recently reported miR-505-5p to be the most informative miRNA of $\mathrm{AD}$ among the OpenArray low abundant miRNAs in CSF samples [24].

A major advantage of the present study is the sample size, which to our knowledge, is the largest sample in an explorative study using large panels of miRNAs to date [5, $24,27,35]$. This large sample size provided adequate power (e.g., power of approximately $80 \%$ or greater) to detect expression differences of 1.2-fold or higher for miRNAs without missing readings and 1.4-fold or higher for miRNAs with missing readings $\left(N_{\text {pairs }}=14\right)$, both moderate effect sizes. Nonetheless, the sample is small compared to many genetic or other epigenetic studies, e.g., DNA methylation studies, and this may be one reason that we only observe nominally significant results. We were, however, able to confirm four of the low-abundant miRNA associations with cognitive scores in the more robust longitudinal setting of repeated assessments (Table 4). Another unique advantage of this study is that it includes monozygotic twin (MZ) pairs. This allowed us to perform both individual and paired analyses, the latter enabling us to cancel out the genetic influence on the phenotype variation and the shared family environment. On the other hand, the intra-pair design has its limitations, particularly with low abundant miRNAs, since both members of a pair needs to have a reading. Thus, we decided in the current work to include only miRNAs that were expressed in at least five complete pairs of twins, and to reach that criterion often miRNAs had to be expressed in a minimum of 20-30 samples. In addition, monozygotic twins are often more similar in terms of cognitive variation compared to the variation between unrelated individuals and even that of dizygotic twin pairs [36]. In the study, we used the Open Array platform that has a disadvantage that only single amplifications were performed, thus the technical variation may be larger than comparable technologies using repeated measures.

In conclusion, our study suggests that several plasma miRNAs may play a role in elderly individual's cognitive aging, including a number of miRNAs that have shared biology with regulation of $\mathrm{AD}$ pathology. In addition, we also observed generally upregulated miRNAs among individuals with poor cognitive health and age-related decline. We recommend that several of our findings should be replicated in future studies of dementia or cognitive aging.

Funding: The Danish Aging Research Center is supported by a grant from the VELUX Foundation. The study was supported by The European Union's Seventh Framework Programme (FP7/2007-2011) under grant agreement $\mathrm{n}^{\circ} 259679$, The Danish Council for Independent Research $(D F F-6110-00016)$ and by Odense University Hospital Free Research Fund.

\section{Compliance with ethical standards}

Conflict of interest The authors declare that they have no conflict of interest.

\section{References}

1. World Health Organization. Dementia. http://www.who.int/media centre/factsheets/fs362/en/, 7 October 2016.

2. Andersen-Ranberg K, Vasegaard L, Jeune B. Dementia is not inevitable: a population-based study of Danish centenarians. J Gerontol B Psychol Sci Soc Sci. 2001;56:P152-9.

3. Danborg PB, Simonsen AH, Waldemar G, Heegaard NH. The potential of microRNAs as biofluid markers of neurodegenerative diseases--a systematic review. Biomarkers. 2014;19:259-68.

4. Cheng L, Quek CY, Sun X, Bellingham SA, Hill AF. The detection of microRNA associated with Alzheimer's disease in biological fluids using next-generation sequencing technologies. Front Genet. 2013;4:150

5. Kumar P, Dezso Z, MacKenzie C, et al. Circulating miRNA biomarkers for Alzheimer's disease. PLoS ONE. 2013;8:e69807.

6. Haqqani AS, Delaney CE, Tremblay TL, Sodja C, Sandhu JK, Stanimirovic DB. Method for isolation and molecular characterization of extracellular microvesicles released from brain endothelial cells. Fluids Barriers CNS. 2013;10:4.

7. Mitchell PS, Parkin RK, Kroh EM, et al. Circulating microRNAs as stable blood-based markers for cancer detection. Proc Natl Acad Sci USA. 2008;105:10513-8.

8. Wu HZ, Ong KL, Seeher K, et al. Circulating microRNAs as biomarkers of alzheimer's disease: a systematic review. J Alzheimers Dis. 2015;49:755-66.

9. Sheinerman KS, Tsivinsky VG, Abdullah L, Crawford F, Umansky SR. Plasma microRNA biomarkers for detection of mild cognitive impairment: biomarker validation study. Aging. 2013;5:925-38.

10. Tan L, Yu JT, Liu QY, et al. Circulating miR-125b as a biomarker of Alzheimer's disease. J Neurol Sci. 2014;336:52-6.

11. Xie B, Zhou H, Zhang R, et al. Serum miR-206 and miR-132 as potential circulating biomarkers for mild cognitive impairment. $\mathrm{J}$ Alzheimers Dis. 2015;45:721-31.

12. Xie B, Liu Z, Jiang L, et al. Increased serum miR-206 level predicts conversion from amnestic mild cognitive impairment to Alzheimer's disease: a 5-year follow-up study. J Alzheimers Dis. 2017;55:509-20.

13. Christensen K, Gaist D, Vaupel JW, McGue M. Genetic contribution to rate of change in functional abilities among Danish twins aged 75 years or more. Am J Epidemiol. 2002;155:132-9. 
14. Skytthe A, Kyvik K, Holm NV, Vaupel JW, Christensen K. The Danish Twin Registry: 127 birth cohorts of twins. Twin Res. 2002;5:352-7.

15. Christiansen L, Frederiksen H, Schousboe K, et al. Age- and sexdifferences in the validity of questionnaire-based zygosity in twins. Twin Res. 2003;6:275-8.

16. McGue M, Christensen K. The heritability of cognitive functioning in very old adults: evidence from Danish twins aged 75 years and older. Psychol Aging. 2001;16:272-80.

17. Hellemans J, Mortier G, De Paepe A, Speleman F, Vandesompele J. qBase relative quantification framework and software for management and automated analysis of real-time quantitative PCR data. Genome Biol. 2017;8:R19.

18. Hellemans J, Vandesompele J. Selection of reliable reference genes for RT-qPCR analysis. Methods Mol Biol. 2014;1160:19-26.

19. Tan Q, Christiansen L, von Bornemann Hjelmborg J, Christensen $\mathrm{K}$. Twin methodology in epigenetic studies. J Exp Biol. 2015;218:134-9.

20. Bates D, Machler M, Bolker BM, Walker SC. Fitting linear mixed-effects models using lme4. J Stat Softw. 2015;67:1-48.

21. Mengel-From J, Thinggaard M, Lindahl-Jacobsen R, McGue M, Christensen K, Christiansen L. CLU genetic variants and cognitive decline among elderly and oldest old. PLoS ONE. 2013;8: e79105.

22. Lee ST, Chu K, Jung KH, et al. miR-206 regulates brain-derived neurotrophic factor in Alzheimer disease model. Ann Neurol. 2012;72:269-77.

23. Sala Frigerio C, Lau P, Salta E, et al. Reduced expression of hsamiR-27a-3p in CSF of patients with Alzheimer disease. Neurology. 2013;81:2103-6.

24. Denk J, Boelmans K, Siegismund C, Lassner D, Arlt S, Jahn H. MicroRNA profiling of CSF reveals potential biomarkers to detect alzheimer's disease. PLoS ONE. 2015;10:e0126423.

25. Coolen M, Katz S, Bally-Cuif L. miR-9: a versatile regulator of neurogenesis. Front Cell Neurosci. 2013;7:220.
26. Schonrock N, Matamales M, Ittner LM, Gotz J. MicroRNA networks surrounding APP and amyloid-beta metabolism--implications for Alzheimer's disease. Exp Neurol. 2012;235:447-54.

27. Pichler S, Gu W, Hartl D, et al. The miRNome of Alzheimer's disease: consistent downregulation of the miR-132/212 cluster. Neurobiol Aging. 2017;50:e1-167.

28. Hancock ML, Preitner N, Quan J, Flanagan JG. MicroRNA-132 is enriched in developing axons, locally regulates Rasa1 mRNA, and promotes axon extension. J Neurosci. 2014;34:66-78.

29. Magill ST, Cambronne XA, Luikart BW, et al. microRNA-132 regulates dendritic growth and arborization of newborn neurons in the adult hippocampus. Proc Natl Acad Sci USA. 2010;107:20382-7.

30. Mengel-From J, Christensen K, McGue M, Christiansen L. Genetic variations in the CLU and PICALM genes are associated with cognitive function in the oldest old. Neurobiol Aging. 2011;32:e7-11.

31. Lindahl-Jacobsen R, Tan Q, Mengel-From J, Christensen K, Nebel A, Christiansen L. Effects of the APOE epsilon2 allele on mortality and cognitive function in the oldest old. J Gerontol A Biol Sci Med Sci. 2013;68:389-94.

32. Schopman NC, Heynen S, Haasnoot J, Berkhout B. A miRNAtRNA mix-up: tRNA origin of proposed miRNA. RNA Biol. 2010;7:573-6.

33. Kim J, Krichevsky A, Grad Y, et al. Identification of many microRNAs that copurify with polyribosomes in mammalian neurons. Proc Natl Acad Sci USA. 2004;101:360-5.

34. Martins M, Rosa A, Guedes LC, et al. Convergence of miRNA expression profiling, alpha-synuclein interacton and GWAS in Parkinson's disease. PLoS ONE. 2011;6:e25443.

35. Schipper HM, Maes OC, Chertkow HM, Wang E. MicroRNA expression in Alzheimer blood mononuclear cells. Gene Regul Syst Bio. 2007;1:263-74.

36. McGue M, Christensen K. Growing old but not growing apart: twin similarity in the latter half of the lifespan. Behav Genet. 2013;43:1-12. 